\title{
State-of-the-Art Treatment and Novel Agents in Local and Distant Recurrences of Cervical Cancer
}

\author{
Clemens B. Tempfer ${ }^{a}$ Matthias W. Beckmann ${ }^{b}$ \\ a Department of Obstetrics and Gynecology, Ruhr University Bochum, Bochum, Germany; \\ ${ }^{b}$ Department of Obstetrics and Gynecology, University of Erlangen, Erlangen, Germany
}

\section{Keywords}

Cervical cancer - Recurrence - Metastatic disease . Treatment

\section{Summary}

Depending on the stage at initial presentation, cervical cancer will recur in $25-61 \%$ of women. Typical manifestations of recurrent cervical cancer include the central pelvis and the pelvic side walls as well as retroperitoneal lymph node basins in the pelvis and the para-aortic region, and - more rarely - supraclavicular lymph nodes. There are no typical symptoms of recurrent cervical cancer. Women with suspected recurrence after cervical cancer based on gynecological examination or organspecific symptoms must undergo imaging studies and if technically feasible - biopsy with histological verification, especially in cases of distant metastases, in order to rule out a second primary. Radiotherapy-naïve women should be treated with salvage radiochemotherapy with curative intention. For women with previous radiotherapy, surgery in the form of hysterectomy, local resection, or pelvic exenteration is the treatment of choice. Pelvic exenteration can lead to cure in selected patients, but at the price of a high rate of complications and significant morbidity and mortality. If complete surgical resection is not feasible or if the woman is not a candidate for surgery, chemotherapy with palliative intent should be offered. Patients with recurrent disease outside the pelvis are candidates for systemic chemotherapy. Several agents have shown to be active in this situation, either in single-agent or combination regimens. Platinumcontaining regimens have a superior efficacy over nonplatinum regimens and bevacizumab may be added to chemotherapy.

(c) 2016 S. Karger GmbH, Freiburg

\section{Introduction}

Cervical cancer is the 7th most common female cancer worldwide and the 16th most common cancer in the European Union [1]. In Germany, the incidence of cervical cancer was 4,600 women in 2012 , with a 5 -year overall survival rate of $61 \%$ [2]. The agestandardized incidence rates of cervical cancer have declined in Germany by $40 \%$ since the 1980 s, but 1,660 women still die from primary advanced or recurrent disease each year [2]. Between 1990 and 2004, the prevalence of cervical cancer declined by $10 \%$, predominantly among women of $>60$ years, whereas the prevalence increased among 40-49-year-old women. In 2004, every second woman with newly diagnosed cervical cancer was $<50$ years old [2]. It is of note that, despite the marked decline in the incidence of cervical cancer during the last 30 years, the 5 -year survival rates remained stable at around 60\% during the periods 1980-1984 and 2000-2004 [2]. This discrepancy may reflect successful cervical cancer screening programs, a general improvement in female health, and changing promiscuity patterns, on the one hand, but also a lack of measurable improvement on the population level regarding the efficacy of primary and recurrent disease treatment during the last 30 years, on the other hand.

Depending on the stage at initial presentation, cervical cancer will recur in $25-61 \%$ of women [3]. In most cases, recurrent disease is diagnosed within 2 years of the initial treatment and may be observed in various sites. Typical manifestations of recurrent cervical cancer include the central pelvis and the pelvic side walls as well as retroperitoneal lymph node basins in the pelvis and the paraaortic region, and - more rarely - supraclavicular lymph nodes. Atypical manifestations of cervical cancer recurrence involve the solid organs of the abdomen as well as the peritoneum, mesentery, and omentum, the gastrointestinal tract, chest, bones, and other sites [4]. For example, in a retrospective analysis of 1,292 women with stage I-IV cervical cancer and definitive radiotherapy, 375 (29\%) had either local or distant failure and 35 patients (3\%) had

\section{KARGER}

(c) 2016 S. Karger GmbH, Freiburg

Fax +497614520714 
both pelvic and distant relapse. Among 162 patients with local failure, 71 had persistent disease and 91 had a relapse after complete initial tumor regression. Among the 213 patients with systemic failure, distant organ metastases were found in $65 \%$ whereas lymphonodular recurrence limited to the para-aortic nodes or to the supraclavicular nodes (with or without para-aortic node involvement) was found in $22 \%$ and $14 \%$, respectively [5]. In another series of 249 patients with stage IB cervical carcinoma treated with radical hysterectomy and pelvic lymphadenectomy, Larson et al. [6] noted recurrent disease in 27 patients (11\%), with pelvic recurrences accounting for 16 of 27 cases (59\%).

There are no typical symptoms of recurrent cervical cancer. Women with lesions involving the vagina may present with symptoms such as discharge, bleeding, dyspareunia, or pain. On gynecological exam, a nodular or solid mass at the vaginal cuff with or without extension to the pelvic side walls may be palpated. Pelvic side wall recurrences often initially present in the form of recurrent urinary infections, urinary retention, and - ultimately - renal failure. Women with systemic recurrence typically present with either no symptoms or non-specific symptoms such as fatigue, nausea, and weight loss. Patients with organ metastases may sometimes present with organ-specific symptoms such as skeletal pain and hemoptysis in case of bone and lung metastases, respectively.

\section{Diagnostic Procedures}

Women with suspected recurrence after cervical cancer based on gynecological examination or organ-specific symptoms should undergo imaging studies and - if technically feasible - biopsy with histological proof of recurrent disease. In women with vaginal recurrence, biopsy is technically easy, but it is more challenging in women with regional or distant recurrence. However, especially isolated metastatic findings on imaging studies may present as a second primary malignancy or a benign process and, thus, histological verification of metastatic disease is important in these cases. This is illustrated by a retrospective study of 21 patients with primary and recurrent cervical cancer who underwent 24 surgical resections for suspicious thoracic metastatic lesions. In this study, Lim et al. [7] noted metastasis from cervical cancer, primary lung cancer, no pathologic abnormality, and tuberculosis in $14,7,2$, and 1 of the surgical specimens, respectively. Different pathologic diagnoses were therefore rendered in 10 of 24 lesions compared to the initial, image-based, diagnosis.

Nowadays, the most commonly used imaging modalities to evaluate recurrent cervical cancer are computed tomography (CT), magnetic resonance imaging (MRI), and positron emission tomography (PET)/CT. PET/CT is the most expensive diagnostic modality, but has a superior sensitivity (93-96\%) and specificity (9395\%) compared to CT and MRI [8]. PET/CT may help distinguish tumor recurrence from a post-therapy residual mass or enlarged reactive lymph nodes, a distinction that is difficult with conventional CT or MRI [9]. In addition, the results of PET/CT scans may alter the management of locally recurrent disease, potentially spar- ing women from an unnecessary, extensive surgical approach in case of additionally detected systemic disease. For example, Kitajima et al. [10] compared PET, CT, and PET/CT in 90 women with histopathologically proven cervical or endometrial cancer recurrence. Patient-based analysis showed that the sensitivity, specificity, and accuracy of PET alone were 79\% (35/44), 74\% (34/46), and $77 \%$ (69/90), respectively, whereas those of CT alone were $68 \%$ (30/44), 87\% (40/46), and 78\% (70/90), respectively, and those of PET/CT were 91\% (40/44), 93\% (43/46), and 92\% (83/90), respectively. PET/CT findings resulted in a change of management in 38 of 90 patients, with an additional effect on patient management in 13 patients diagnosed by $\mathrm{CT}$ alone and in 14 patients diagnosed by PET alone.

However, it should be noted that, while PET/CT may be used in symptomatic women with suspected cervical cancer recurrence, the routine use of PET/CT for the surveillance of asymptomatic patients after the completion of primary and adjuvant treatment is controversial because of the low true-positive rate of $44 \%$ in asymptomatic patients compared with $71 \%$ in symptomatic patients [9]. Thus, current guidelines, although acknowledging the potential value of PET/CT in the diagnostic work-up of women with recurrent disease, do not endorse the routine use of this modality during posttreatment surveillance of women after completion of the initial treatment [11].

\section{Treatment of Local Pelvic Recurrence}

Local recurrence may occur in the cervix, the vagina, the central pelvis, or the pelvic side walls. In some of these women, a curative treatment approach is possible. Criteria to identify these women are isolated, localized disease, resectable disease, and/or no previous radiotherapy. The specific choice of therapy depends on the previous treatment modalities. Specifically, there are 2 groups of women: those who have previously undergone primary radiochemotherapy or adjuvant radiochemotherapy during their firstline treatment and those who are radiotherapy naïve, i.e. who have not undergone any form of radiotherapy during first-line treatment. For women who have already undergone radiotherapy, surgery in the form of hysterectomy, local resection, or pelvic exenteration is the treatment of choice, if possible. If complete surgical resection is not possible or if the woman is not a candidate for surgery, chemotherapy with palliative intent should be offered. The approach to these patients is then identical to the treatment of women with metastatic disease. The second group of women, i.e. those who are radiotherapy naive, should be treated with salvage radiochemotherapy with curative intent. The specifics of these treatments are discussed below.

\section{Surgical Treatment}

Women with local recurrence in the cervix and vagina and those with a central pelvic recurrence after initial radiochemotherapy or after initial radical hysterectomy followed by adjuvant radiochemotherapy may be treated with curative surgery. Surgical 
therapy in these women consists of local resection in rare cases of limited vaginal recurrence, hysterectomy in case of a cervical recurrence, or pelvic exenteration. There are three forms of pelvic exenteration: total, anterior, and posterior pelvic exenteration. Total pelvic exenteration involves the resection of the tumor, the uterus (if still present), the vagina, the bladder, and the rectum, in association with colostomy creation and a ureteral stoma, mostly a non-continent ileal urinary conduit. Anterior pelvic exenteration involves the resection of the tumor, the uterus (if present), the vagina, and the bladder, with a ureteral stoma. Posterior exenteration involves the resection of the tumor, the uterus (if present), the vagina, and the rectum, with colostomy creation. The 5-year survival rates of patients who undergo total pelvic exenteration range from 20 to $60 \%$ [12-14]. Clearly, exenteration is a major surgical procedure and is associated with a procedure-associated mortality of up to $10 \%$ [12-18]. Therefore, extensive counselling of patients is necessary before undergoing this treatment. Another problem with exenteration is that, despite extensive preoperative diagnostic work-up to judge resectability and to exclude distant metastases, between 30 and $50 \%$ of cases are found to be unresectable at the time of laparotomy due to peritoneal spread, para-aortic node involvement, or pelvic side wall involvement $[15,16]$. Therefore, the surgical goal of exenterative surgery - complete tumor resection cannot be achieved in a major proportion of patients undergoing this procedure. For example, Fotopoulou et al. [16] achieved a complete tumor resection in only 23/47 women undergoing exenteration. Andikyan et al. [17] report on 11 patients who underwent anterior exenteration for recurrent gynecological malignancies (cervix, 6; uterus, 3; vagina, 1; and urethra, 1). Complete surgical resection with negative pathologic margins was achieved in all 11 patients, with major postoperative complications occurring in 4 patients (36\%). With a median follow-up after the procedure of 25 months (range 6-95 months), none of the patients developed a pelvic recurrence. It is, however, of note that the median size of the recurrent tumor in this series was only $0.9 \mathrm{~cm}$ (range, microscopic to $4 \mathrm{~cm}$ ), suggesting surgical overtreatment in at least some of these cases. Criteria to identify women most likely to benefit from exenterative surgery have been proposed as follows [18]:

- a central pelvic recurrence without side wall fixation or associated hydronephrosis,

- a long disease-free interval since primary treatment, and

- a tumor size of the recurrence of less than $3 \mathrm{~cm}$ in diameter.

These criteria, however, question the concept of exenteration, since it is difficult to argue such a morbid procedure for women with recurrent disease measuring a few centimeters. In addition, there is no randomized clinical trial establishing the superiority of pelvic exenteration over systemic chemotherapy or best supportive care. Despite a number of case series reporting favorable surgical outcomes of women after pelvic exenteration, a reliable assessment of the efficacy of this treatment approach is not possible due to heterogeneous study results, patient selection, and publication bias. A systematic review of the literature of the Cochrane Collaboration identified no randomized trial and concluded that there is no evidence to inform decisions about exenterative surgery for women with recurrent gynecologic malignancies [19]. Sardain et al. [18] performed pelvic exenterations in 13 women with recurrent cervical cancer and 3 women with recurrent vaginal cancer. All of the patients had received pelvic irradiation before; the median tumor size was $4.3 \mathrm{~cm}$. There were no intraoperative or postoperative deaths and the 5-year disease-free survival and overall survival rates were $30 \%$ and $34 \%$, respectively. In contrast, Capilna et al. [20] reported in-hospital complications in 6 patients (40\%) with 2 perioperative deaths (13\%) in a series of 15 exenterations (6 total, 4 anterior, and 5 posterior). 6 patients died during the follow-up. Terán-Porcayo et al. [21] performed 42 pelvic exenterations for post-radiotherapy recurrent or persistent cervical cancer. Intraand postoperative complications were observed in $65 \%$ of the patients, with a surgical mortality of $5 \%$. Survival at 5 years was $66 \%$. Marnitz et al. [22] reported on a series of 55 patients who underwent exenterative surgery. They performed primary exenterative surgery in 20 patients with stage IVA cervical cancer and secondary exenterative surgery in 35 patients with recurrent cervical cancer. The overall cumulative survival at 5 years was $53 \%$ in the primary group and only $27 \%$ in the recurrent group. Complications were noted in $57 \%$ of the patients, most commonly fistulas and gastrointestinal complications. The operative surgery-associated mortality was $6 \%$. Survival correlated with the time interval between primary treatment and recurrence as well as with tumor-free resection margins (2-year survival rate of $10 \%$ for positive margins compared to a 5 -year survival rate of $55 \%$ for negatives margins).

Some patients treated with pelvic exenteration may benefit from additional intraoperative radiation therapy. However, the efficacy of intraoperative radiation therapy in conjunction with pelvic exenteration has not been demonstrated in prospective trials [23]. In summary, these data show that pelvic exenteration can lead to cure in selected patients with postradiotherapy local recurrence, but at the price of a high rate of complications and a significant longterm morbidity and mortality. The lack of controlled trials and the fact that optimal results of exenterative procedures have been reported for lesion sizes ranging from microscopic disease to $4 \mathrm{~cm}$ $[16,17]$ question the whole concept. Thus, an individualized management with careful selection and counselling of the patients is warranted $[19,24]$.

\section{Radiotherapy}

Women who are radiotherapy naïve may be treated with salvage radiochemotherapy with curative intent. In addition, radiochemotherapy is a reasonable option for women with local recurrence and a history of radical hysterectomy and brachytherapy alone. Given the superiority of radiochemotherapy over radiotherapy in the primary treatment setting, most experts prefer salvage combination radiochemotherapy for patients with recurrent disease [25, 26]. However, studies published in the literature have used both radiotherapy and radiochemotherapy in this situation. For example, the benefit of salvage radiotherapy was demonstrated in a single-institution experience of 35 women who were treated with high-dose radiotherapy following a local pelvic recurrence [25]. After a median follow-up period of 12 years, the 5- and 10-year 
Table 1. Response rates to salvage chemoradiation in women with cervical cancer recurrence according to the site of recurrence (from [26] with permission)

\begin{tabular}{|c|c|c|c|c|c|}
\hline \multirow[t]{2}{*}{ Site of recurrence } & \multicolumn{5}{|c|}{ Response to treatment, $\mathrm{n}(\%)$} \\
\hline & CR & PR & SD & DP & Total \\
\hline \multicolumn{6}{|l|}{ Pelvis } \\
\hline Central & $15(71)$ & $3(14)$ & $2(10)$ & $1(5)$ & $21(45)$ \\
\hline Peripheral & $8(62)$ & $5(38)$ & - & - & $13(28)$ \\
\hline Para-aortic & $6(75)$ & $1(13)$ & - & $1(13)$ & $8(17)$ \\
\hline Multiple & $3(75)$ & - & $1(25)$ & - & $4(9)$ \\
\hline Abdominal wall & $1(100)$ & - & - & - & $1(2)$ \\
\hline Total & $33(70)$ & $9(19)$ & $3(6)$ & $2(4)$ & 47 \\
\hline
\end{tabular}

$\mathrm{CR}=$ Complete response, $\mathrm{PR}=$ partial response, $\mathrm{SD}=$ stable disease,

$\mathrm{DP}=$ disease progression.

Because of rounding, not all percentages total 100 .

overall survival rates were $43 \%$ and $33 \%$, respectively. The pelvic control rates at 5 and 10 years were $69 \%$ and $62 \%$, respectively. The use of brachytherapy and a long treatment-free interval between primary surgery and the diagnosis of recurrence were positive predictors of a good outcome. Salvage radiochemotherapy has been used effectively by Lee et al. [26] in 47 women with recurrent cervical cancer, using a median dose of $65 \mathrm{~Gy}$, including an additional brachytherapy boost in 10 of the patients. Grade 3-4 acute hematologic toxicity was observed in 29 (62\%) women. The 5-year cumulative incidence of severe gastrointestinal and genitourinary toxicity was $13 \%$ and $7 \%$, respectively. 33 patients $(70 \%)$ showed a complete response and $9(19 \%)$ a partial response. Table 1 shows the response rates according to the site of recurrence. In this study, the 5 -year overall and disease-free survival rates were $44 \%$ and $41 \%$, respectively.

Radiochemotherapy can also be used for women with isolated lymph node recurrence after cervical cancer. For example, Kim et al. [27] treated 12 patients with isolated para-aortic lymph node recurrence. The fractionated dose was $1.2 \mathrm{~Gy}$ in 2 daily fractions, and the median total dose was $60 \mathrm{~Gy}$. The weekly concurrent chemotherapy consisted of paclitaxel in 11 patients and cisplatin in 1 patient. The median number of cycles of chemotherapy was 5 . A complete tumor response was noted in $33 \%(4 / 12)$ and a partial response in $67 \%(8 / 12)$. The 3 -year overall survival rate and the median survival duration were $19 \%$ and 21 months, respectively. However, the outcome of women with isolated lymph node recurrence is poor, irrespective of the treatment modality. This was illustrated in another retrospective study of 375 patients with recurrent cervical cancer, of whom 60 had limited metastatic disease involving the para-aortic nodes and 26 had limited metastatic disease involving the supraclavicular nodes. The overall survival at 5 years was $27 \%$ and $0 \%$, respectively [5].

\section{Intraoperative Radiotherapy and Laterally Extended Endopelvic} Resection

Laterally extended endopelvic resection (LEER) is a surgical procedure designed to improve locoregional tumor control by de- lineating surgery margins according to the ontogenetic anatomy. Höckel et al. [28] reported a series of 91 patients with locally advanced primary $(n=30)$ and recurrent or persistent $(n=61)$ carcinoma of the cervix and vagina who were treated with LEER, most of them fixed to the pelvic side walls. Of note, complete resection with free margins was histopathologically confirmed in all cases. LEER definitively controlled the locoregional cancer in $92 \%$ of the patients, with a 5 -year overall survival rate of $61 \%$. These data suggest that LEER may be an additional treatment option for women with pelvic side wall recurrences, who are normally considered candidates for irradiation. Other institutions have adopted the LEER procedure with or without the addition of intraoperative radiotherapy (IORT). For example, Backes et al. [29] reported a series of 32 previously irradiated women with a pelvic recurrence from cervical, vaginal, and vulvar cancer. Of these patients, 22 did and 10 did not receive IORT in addition to pelvic exenteration or LEER. The median progression-free and overall survival times were 10 and 10 months for pelvic exenteration + IORT compared to 9 and 17 months for LEER + IORT. Of note, no patients undergoing LEER + IORT recurred locally, whereas $62 \%$ recurred with a distant component. In contrast, patients with exenteration alone had mainly local recurrences compared to equally distributed local and distant recurrences for those with exenteration + IORT. This suggests that the best results are achieved if the tumor is completely resectable, whereas IORT may select patients with residual disease and the subsequent need for IORT.

In another case series, Barney et al. [23] treated 86 patients with locally recurrent $(n=73)$ or primary advanced $(n=13)$ cervical cancer with IORT (electron beam therapy, median dose $15 \mathrm{~Gy}$ ) following surgery. The surgery types were pelvic exenteration or pelvic side wall resection. 61 patients received additional perioperative external beam radiotherapy (EBRT) with a median dose of $45 \mathrm{~Gy}$. In this series, $\mathrm{R} 0$ resections were achieved in $41 \%$ of cases $(\mathrm{n}=35)$, and $\mathrm{R} 1$ and $\mathrm{R} 2$ resections in $35 \%(\mathrm{n}=30)$ and $24 \%(\mathrm{n}=21)$ of cases, respectively. Cumulative incidences of central relapses within the irradiated field and locoregional relapse at 3 years were $23 \%$ and $38 \%$, respectively. The 3 -year cumulative incidence of distant relapse was $43 \%$. The median survival was 15 months. On multivariate analysis, pelvic exenteration $(\mathrm{p}=0.02)$ and perioperative EBRT ( $p=0.009$ ) were associated with improved central control in patients with recurrent disease. Common IORT-related toxicities included peripheral neuropathy $(\mathrm{n}=16)$, ureteral stenosis $(\mathrm{n}=4)$, and bowel fistula/perforation $(\mathrm{n}=4)$.

In summary, these data demonstrate that both LEER and IORT alone or in combination are feasible and may achieve good longterm outcomes in patients with pelvic recurrence from cervical cancer. However, LEER requires a high level of expertise and IORT adds toxicity to a surgical procedure already associated with substantial morbidity. Thus, both techniques require careful patient selection.

\section{Conclusions}

Long-term survival is possible with combined modality therapy including IORT for advanced cervical cancer. Distant relapse is 
Table 2. Objective response to 4 different chemotherapy regimens in women with cervical cancer recurrence (from [33] with permission)

\begin{tabular}{|c|c|c|c|c|c|c|c|c|c|}
\hline \multirow[t]{2}{*}{ Tumor response } & \multicolumn{2}{|c|}{$\mathrm{Cis}+\mathrm{Pac}$} & \multicolumn{2}{|c|}{ Cis + Vin } & \multicolumn{2}{|c|}{ Cis + Gem } & \multicolumn{2}{|c|}{ Cis + Top } & \multirow[b]{2}{*}{ Total } \\
\hline & $\mathrm{n}$ & $\%$ & $\mathrm{n}$ & $\%$ & $\mathrm{n}$ & $\%$ & $\mathrm{n}$ & $\%$ & \\
\hline Responders & 30 & 29.1 & 28 & 25.9 & 25 & 22.3 & 26 & 23.4 & 109 \\
\hline Complete & 3 & 2.9 & 8 & 7.4 & 1 & 0.9 & 2 & 1.8 & 14 \\
\hline Partial & 27 & 26.2 & 20 & 18.5 & 24 & 21.4 & 24 & 21.6 & 95 \\
\hline Stable disease & 50 & 48.4 & 46 & 42.6 & 54 & 48.2 & 53 & 47.8 & 203 \\
\hline $\begin{array}{l}\text { Progressive } \\
\quad \text { disease/other }\end{array}$ & 23 & 22.3 & 34 & 31.5 & 33 & 29.5 & 32 & 28.8 & 122 \\
\hline Total & \multicolumn{2}{|l|}{103} & \multicolumn{2}{|l|}{108} & \multicolumn{2}{|l|}{112} & \multicolumn{2}{|l|}{111} & 434 \\
\hline Odds ratio ${ }^{\mathrm{a}}$ & \multicolumn{2}{|c|}{-} & \multicolumn{2}{|c|}{1.17} & \multicolumn{2}{|c|}{1.43} & \multicolumn{2}{|c|}{1.34} & \\
\hline $95 \% \mathrm{CI}^{\mathrm{b}}$ & \multicolumn{2}{|c|}{ - } & \multicolumn{2}{|c|}{$0.54-2.58$} & \multicolumn{2}{|c|}{$0.65-3.19$} & \multicolumn{2}{|c|}{$0.61-2.98$} & \\
\hline
\end{tabular}

Cis + Pac $=$ Cisplatin + paclitaxel, Cis + Vin $=$ cisplatin + vinorelbine, Cis $+\mathrm{Gem}=$ cisplatin + gemcitabine, $\mathrm{Cis}+\mathrm{Top}=$ cisplatin + topotecan, $\mathrm{CI}=$ confidence interval.

${ }^{a}$ Odds ratios for response are provided for the reference arm, Cis + Pac, to the experimental therapies. ${ }^{b} \mathrm{CIs}$ are adjusted with a Bonferroni correction. common; yet, a significant number of patients experience local progression in spite of aggressive treatment. In addition to consideration of disease- and treatment-related morbidity, other factors to be considered when selecting patients for this approach include the time interval between initial therapy and recurrence and whether the patient is able to receive perioperative EBRT and pelvic exenteration or LEER in addition to IORT.

\section{Treatment of Distant Recurrence}

Patients with recurrent disease outside the pelvis are candidates for systemic chemotherapy. Several agents have been shown to be active in recurrent cervical cancer, either as single-agent therapy or as part of a combination regimen. For example, the effectiveness of gemcitabine, paclitaxel, vinorelbine, and camptothecines has been tested in phase II studies. When used as single agents, these compounds have an overall response rate (ORR) between 8 and 25\% $[30,31]$. Interestingly, cisplatin-containing regimens have a superior efficacy over non-platinum regimens, with ORRs between 41 and $60 \%$ [30], despite the fact that many patients have received cisplatin as part of the adjuvant radiochemotherapy. Most experts therefore recommend cisplatin either as a single agent or in combination with another compound, e.g. topotecan, for patients with metastatic recurrent cervical cancer, irrespective of a potential previous treatment with cisplatin as a radiosensitizer [31]. It has to be noted, however, that there are no head-to-head comparisons in randomized trials comparing single-agent cisplatin with other single agents (e.g. paclitaxel, gemcitabine, or vinorelbine).

In general, combination chemotherapy should be preferred over single-agent regimens when treating patients with recurrent cervical cancer. This recommendation is based on a Cochrane metaanalysis of 5 randomized trials with 1,114 patients [32]. Compared with combination platinum-based therapy, single-agent cisplatin had a lower response rate (relative risk (RR) $0.60,95 \%$ confidence interval (CI) $0.44-0.81$ ), but was associated with less toxicity, in-

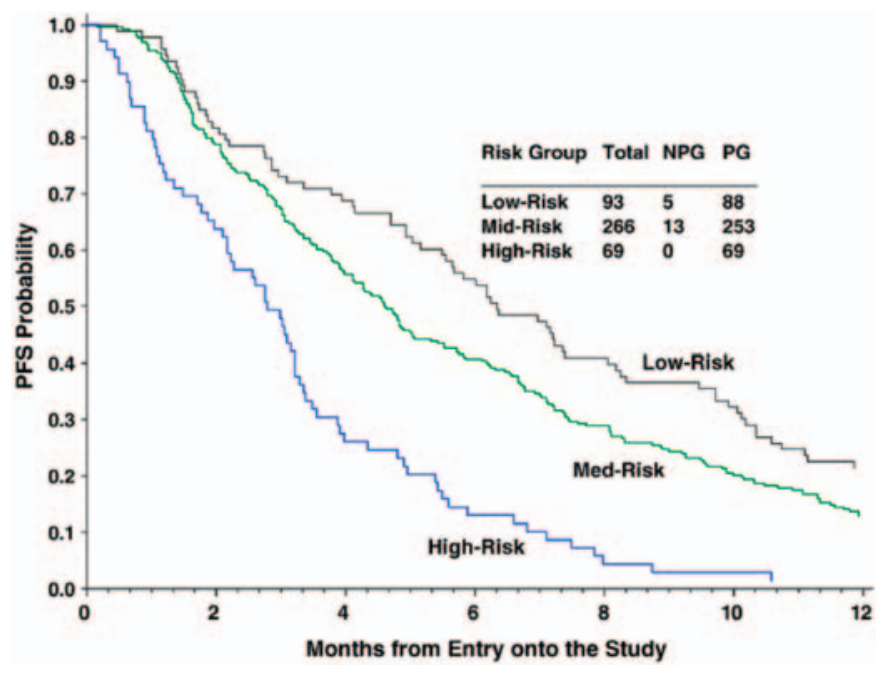

Fig. 1. Kaplan-Meier curves of progression-free survival (PFS) stratified by the number of risk factors being present in 3 patient groups (low-risk $=0-1$ risk factors; mid-risk $=2-3$ risk factors; high-risk $=4-5$ risk factors) (from [31] with permission).

cluding less serious neutropenia (RR 0.04, 95\% CI 0.02-0.12) and thrombocytopenia (RR $0.16,95 \%$ CI $0.05-0.48$ ). Various combination chemotherapy regimens are available and seem to achieve comparable results. This has been demonstrated by the Gynecologic Oncology Group (GOG) study 204 with 434 patients with metastatic or advanced cervical cancer [33]. Patients were randomly assigned to cisplatin plus paclitaxel as the reference regimen or 1 of the experimental regimens (cisplatin plus vinorelbine, cisplatin plus gemcitabine, or cisplatin plus topotecan). Interestingly, there were no significant differences regarding the response rates (table 2). Cisplatin and paclitaxel produced an ORR of $29 \%$, whereas the ORR was $26 \%, 22 \%$, and $23 \%$ for the other 3 regimens, respectively. As expected, side effects were different between the regimens, with cisplatin/paclitaxel causing less thrombocytopenia and cisplatin/gemcitabine causing less febrile neutropenia than the other regimens. 
Fig. 2. Kaplan-Meier curves of overall and progression-free survival in women with cervical cancer recurrence and chemotherapy with carboplatin/ paclitaxel (from [32] with permission). paclitaxel and cisplatin/

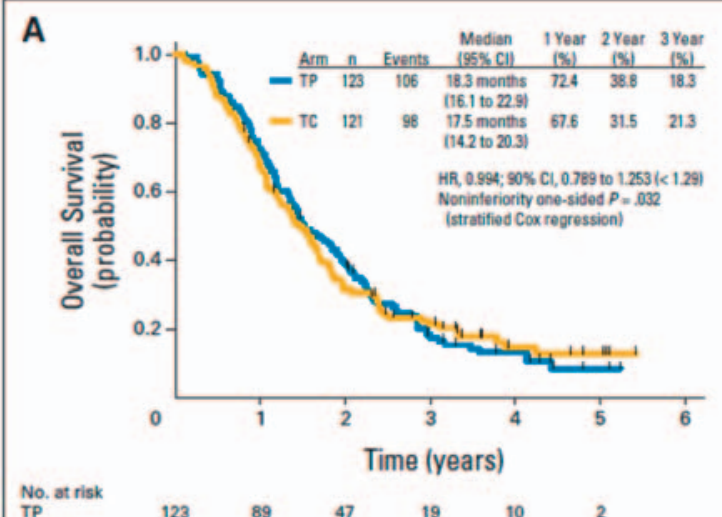

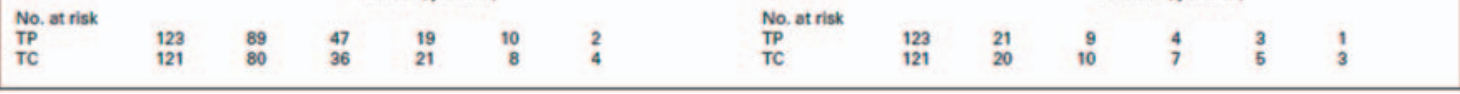

A

Fig. 3. Kaplan-Meier curves of overall and progression-free survival in women with cervical cancer recurrence and chemotherapy with and without bevacizumab (from [34] with permission).

B
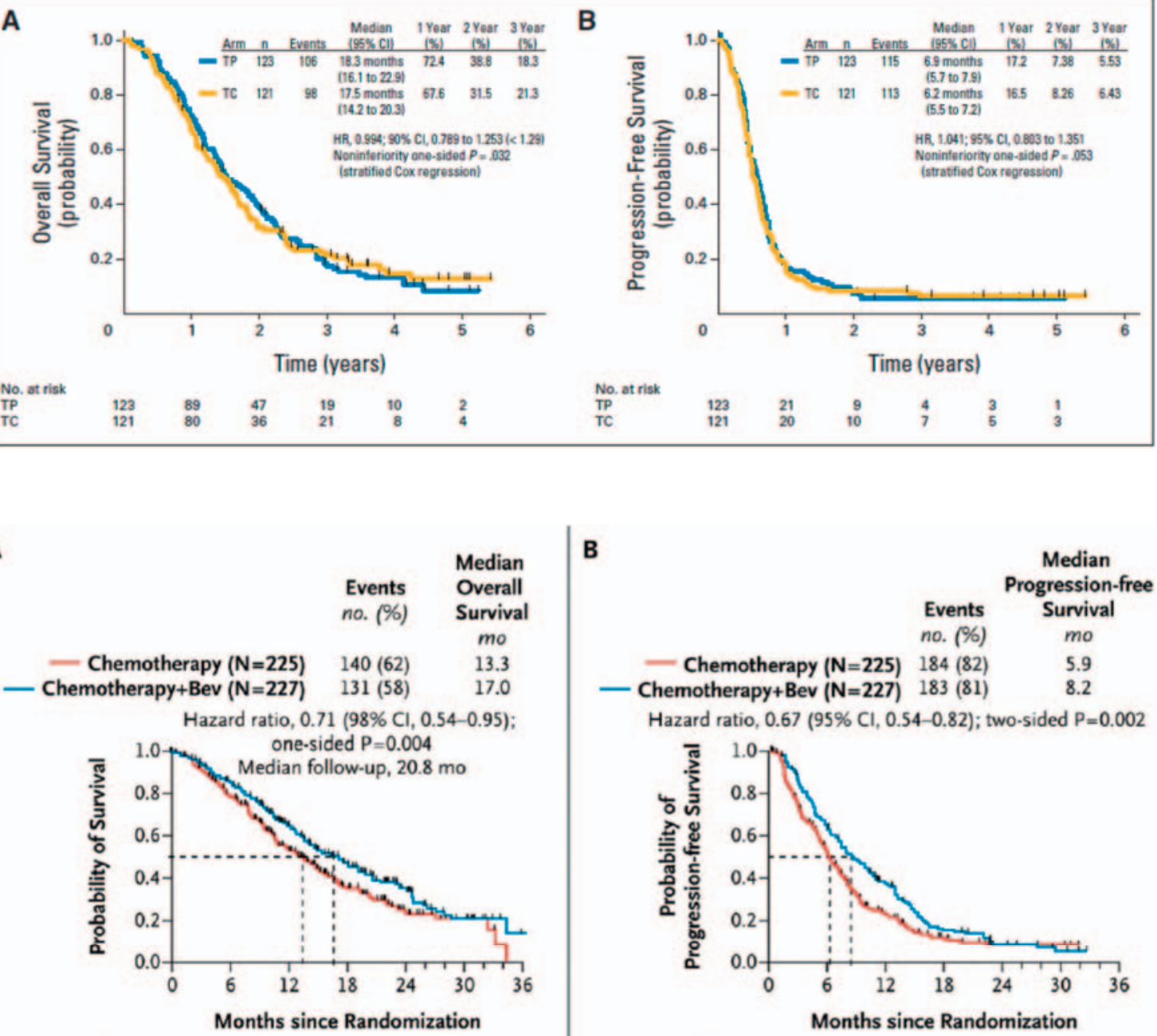

No. at Risk

$\begin{array}{lllllll}\text { Chemotherapy } & 225 & 167 & 94 & 45 & 17 & 8\end{array}$ $\begin{array}{lllllll}\text { Chemotherapy } & 227 & 184 & 121 & 69 & 30 & 10\end{array}$
B

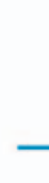

Events
no. (\%)
Chemotherapy (N=225) $184(82)$

Median

Progression-free

Survival

- Chemotherapy+Bev $(\mathrm{N}=227) \quad 183(81) \quad 8.2$

Hazard ratio, 0.67 (95\% Cl, 0.54-0.82); two-sided $\mathrm{P}=0.002$

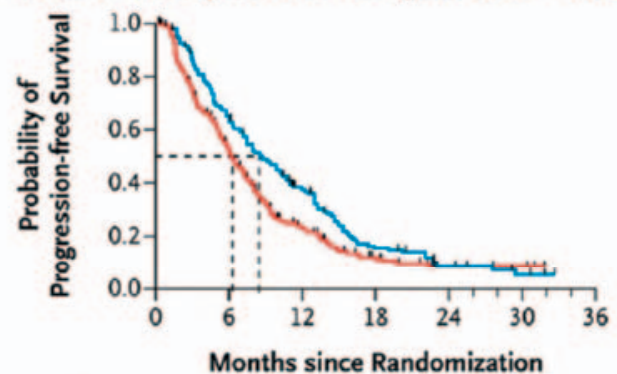

No. at Risk

$\begin{array}{lllllll}\text { Chemotherapy } & 225 & 103 & 40 & 14 & 6 & 3\end{array}$

$\begin{array}{lllllll}\text { Chemotherapy } & 227 & 132 & 70 & 22 & 6 & 3\end{array}$
Although women who have undergone previous cisplatin-containing radiochemotherapy have a better response to cisplatin compared to those having received other agents, they still have a poorer response compared to chemotherapy-naïve women. In addition, response to chemotherapy depends on a number of other factors. For example, in a retrospective cohort study of 428 patients with metastatic cervical cancer, the response to chemotherapy was assessed and independent predictors of response were identified [34]. In this study, African-American race, performance status $>0$, pelvic disease, prior radiosensitizer therapy, and a time interval from diagnosis to first recurrence of $<1$ year independently predicted a poor response. Specifically, patients with a combination of these risk factors had a response rate of only $13 \%$ and a median progression-free and overall survival of only 2.8 and 5.5 months, respectively. Figure 1 shows the progression-free survival curves stratified by the number of risk factors being present.

In order to reduce the side effects of cisplatin, this substance may be safely replaced by carboplatin. This has been successfully demonstrated by the Japanese Clinical Oncology Group 0505 trial [35]. In this study, 253 women with stage IVB, persistent or recurrent cervical cancer were randomly assigned to cisplatin $(50 \mathrm{mg}$ / $\mathrm{m}^{2}$ ) plus paclitaxel $\left(135 \mathrm{mg} / \mathrm{m}^{2}\right)$ or carboplatin (area under the curve (AUC) 5) plus paclitaxel $\left(175 \mathrm{mg} / \mathrm{m}^{2}\right)$. Prior cisplatin therapy was noted in $43 \%$ and $50 \%$ of each group. Carboplatin/paclitaxel was non-inferior to cisplatin/paclitaxel, with a median overall survival duration of 17.5 months for carboplatin/paclitaxel versus 18.3 months for cisplatin/paclitaxel (fig. 2). As expected, carboplatin/paclitaxel was better tolerated, with significantly less serious neutropenic events, renal insufficiency, nausea, and vomiting. Of note, patients who had not received prior cisplatin, benefited more from cisplatin/paclitaxel, with an overall survival duration of 23 versus 13 months, suggesting that carboplatin should only be used in women with prior radiotherapy. In summary, metastatic disease in women with cervical cancer recurrence should be treated with a combination chemotherapy containing cisplatin or carboplatin. 


\section{Novel Substances}

Targeted therapies have been introduced in the treatment of recurrent cervical cancer. The most prominent substance and the only one implemented into clinical practice so far is bevacizumab. Bevacizumab is an expensive drug, and the addition of bevacizumab to chemotherapy is not cost-effective in this setting [36]. However, it has been shown to improve the overall survival duration by 4 months [37]. The GOG 240 study randomized 452 patients to chemotherapy (cisplatin $50 \mathrm{mg} / \mathrm{m}^{2}$ and paclitaxel 135 or $175 \mathrm{mg} / \mathrm{m}^{2}$ or topotecan $0.75 \mathrm{mg} / \mathrm{m}^{2}$ and paclitaxel $175 \mathrm{mg} / \mathrm{m}^{2}$ ) with or without bevacizumab at a dose of $15 \mu \mathrm{g}$ per kilogram of body weight. With the data for the 2 chemotherapy regimens combined, the addition of bevacizumab to chemotherapy significantly increased overall survival (17.0 vs. 13.3 months) (fig. 3) and produced higher response rates ( $48 \%$ vs. $36 \%$ ). Bevacizumab, as compared to chemotherapy alone, was associated with an increased incidence of hypertension ( $25 \%$ vs. $2 \%$ ), thromboembolic events ( $8 \%$ vs. $1 \%$ ), and gastrointestinal fistulas (3\% vs. $0 \%$ ). In August 2014, bevacizumab received approval for cervical cancer recurrence in the USA by the US Food and Drug Administration.

Other targeted therapies have not yet reached the clinic. One candidate is cetuximab, which has been tested in several trials. For example, a randomized phase II trial enrolled 78 FIGO (Fédération Internationale de Gynécologie et d'Obstétrique) stage IB2-IIIB cervical cancer patients to either cisplatin-based radiochemotherapy alone or conjointly with a 6-week course of weekly cetuximab. The result of this study, however, was disappointing because cetuximab did not improve the disease-free survival at 24 months.

\section{Conclusions}

The treatment of cervical cancer recurrence is challenging. Women with suspected recurrence after cervical cancer based on gynecological examination or organ-specific symptoms should undergo imaging studies such as CT, MRI or PET/CT and - if technically feasible - biopsy with histological proof of recurrent disease. Radiotherapy-naïve women should be treated with salvage radiochemotherapy with curative intent. For women who have previously undergone radiotherapy, surgery in the form of hysterectomy, local resection, or pelvic exenteration is the treatment of choice, if possible. Pelvic exenteration, however, has a high rate of complications and significant morbidity and mortality. If complete surgical resection is not possible or if the woman is not a candidate for surgery, chemotherapy with palliative intent should be offered. Patients with recurrent disease outside the pelvis are candidates for systemic chemotherapy. Several agents have shown to be active in recurrent cervical cancer, either as single-agent therapy or as part of a combination regimen. Platinum-containing regimens have a superior efficacy over non-platinum regimens. Bevacizumab prolongs the duration of survival and may be added to chemotherapy.

\section{Disclosure Statement}

The authors declare that they have no conflict of interest.

\section{References}

1 Ferlay J, Steliarova-Foucher E, Lortet-Tieulent J, et al.: Cancer incidence and mortality patterns in Europe: estimates for 40 countries in 2012. Eur J Cancer 2013;49: 1374-1403.

2 Robert Koch-Institut (Hrsg): Verbreitung von Krebserkrankungen in Deutschland. Entwicklung der Prävalenzen zwischen 1990 und 2010. Beiträge zur Gesundheitsberichterstattung des Bundes. Berlin, RKI, 2010.

3 Ries LAG, Harkins D, Krapcho M, et al.: SEER Cancer Statistics Review, 1975 to 2003. Bethesda, National Cancer Institute, 2006.

4 Fulcher AS, O'Sullivan SG, Segreti EM, et al.: Recurrent cervical carcinoma: typical and atypical manifestations. Radiographics 1999;19:264-265.

5 Hong JH, Tsai CS, Lai CH, et al.: Recurrent squamous cell carcinoma of cervix after definitive radiotherapy. Int J Radiat Oncol Biol Phys 2004;60:249-257.

6 Larson DM, Copeland LJ, Stringer CA, et al.: Recurrent cervical carcinoma after radical hysterectomy. Gynecol Oncol 1988;30:381-387.

7 Lim MC, Lee HS, Seo SS, et al.: Pathologic diagnosis and resection of suspicious thoracic metastases in patients with cervical cancer through thoracotomy or video-assisted thoracic surgery. Gynecol Oncol 2010; 116:478-482.

8 Patel CN, Nazir SA, Khan Z, et al.: 18F-FDG PET/CT of cervical carcinoma. AJR Am J Roentgenol 2011;196: 1225.
Mittra E, El-Maghraby T, Rodriguez CA, et al.: Efficacy of 18F-FDG PET/CT in the evaluation of patients with recurrent cervical carcinoma. Eur J Nucl Med Imaging 2009;36:1952-1959.

10 Kitajima K, Murakami K, Yamasaki E, et al.: Performance of integrated FDG-PET/contrast-enhanced CT in the diagnosis of recurrent uterine cancer: comparison with PET and enhanced CT. Eur J Nucl Med Imaging 2009;36:362-372.

11 S3-Leitlinie Diagnostik, Therapie und Nachsorge der Patientin mit Zervixkarzinom, Version 1.0 - September 2014, AWMF-Registernummer 032/033OL. www.awmf. org/uploads/tx_szleitlinien/032-033OLl_S3_Zervixkarzinom_2014-10.pdf, 2014 [last accessed March 27, 2016].

12 Rutledge FN, Smith JP, Wharton JT, et al.: Pelvic exenteration: analysis of 296 patients. Am J Obstet Gynecol 1977;129:881-892.

13 Matthews CM, Morris M, Burke TW, et al.: Pelvic exenteration in the elderly patient. Obstet Gynecol 1992; 79:773-777.

14 Symmonds RE, Pratt JH, Webb MJ: Exenterative operations: experience with 198 patients. Am J Obstet Gynecol 1975;121:907-918.

15 Estape R, Angioli R: Surgical management of advanced and recurrent cervical cancer. Semin Surg Oncol 1999; 16:236-241.

16 Fotopoulou C, Neumann U, Kraetschell R, et al.: Longterm clinical outcome of pelvic exenteration in patients with advanced gynecological malignancies. J Surg Oncol 2010;101:507-512.
17 Andikyan V, Khoury-Collado F, Gerst SR, et al.: Anterior pelvic exenteration with total vaginectomy for recurrent or persistent genitourinary malignancies: review of surgical technique, complications, and outcome. Gynecol Oncol 2012;126:346-350.

18 Sardain H, Lavoué V, Laviolle B, et al.: Prognostic factors for curative pelvic exenterations in patients with recurrent uterine cervical or vaginal cancer. Int J Gynecol Cancer 2014;24:1679-1685.

19 Ang C, Bryant A, Barton DP, et al.: Exenterative surgery for recurrent gynaecological malignancies. Cochrane Database Syst Rev 2014;(2):CD010449.

20 Capilna ME, Moldovan B, Szabo B: Pelvic exenteration - our initial experience in 15 cases. Eur J Gynaecol Oncol 2015;36:142-145.

21 Terán-Porcayo MA, Zeichner-Gancz I, del-Castillo RA, Beltrán-Ortega A, Solorza-Luna G: Pelvic exenteration for recurrent or persistent cervical cancer: experience of five years at the National Cancer Institute in Mexico. Med Oncol 2006;23:219-223.

22 Marnitz S, Köhler C, Müller M, et al.: Indications for primary and secondary exenterations in patients with cervical cancer. Gynecol Oncol 2006;103:1023-1030.

23 Barney BM, Petersen IA, Dowdy SC, Bakkum-Gamez JN, Klein KA, Haddock MG: Intraoperative electron beam radiotherapy (IOERT) in the management of locally advanced or recurrent cervical cancer. Radiat Oncol 2013;8:80 
24 Friedlander M, Grogan M; U.S. Preventative Services Task Force: Guidelines for the treatment of recurrent and metastatic cervical cancer. Oncologist 2002;7:342-347.

25 Haasbeek CJ, Uitterhoeve AL, van der Velden J, et al. Long-term results of salvage radiotherapy for the treatment of recurrent cervical carcinoma after prior surgery. Radiother Oncol 2008;89:197-204.

26 Lee YS, Kim YS, Kim JH, et al.: Feasibility and outcome of concurrent chemoradiotherapy for recurrent cervical carcinoma after initial surgery. Tumori 2010; 96:553-559.

27 Kim JS, Kim JS, Kim SY, et al.: Hyperfractionated radiotherapy with concurrent chemotherapy for paraaortic lymph node recurrence in carcinoma of the cervix. Int J Radiat Oncol Biol Phys 2003;55:1247-1253.

28 Höckel M, Horn LC, Einenkel J: (Laterally) Extended endopelvic resection: surgical treatment of locally advanced and recurrent cancer of the uterine cervix and vagina based on ontogenetic anatomy. Gynecol Oncol 2012;127:297-302.
29 Backes FJ, Billingsley CC, Martin DD, Tierney BJ, Eisenhauer EL, Cohn DE, O’Malley DM, Salani R, Copeland LJ, Fowler JM: Does intra-operative radiation at the time of pelvic exenteration improve survival for patients with recurrent, previously irradiated cervical, vaginal, or vulvar cancer? Gynecol Oncol 2014;135:95-99.

30 Savarese A, Cognetti F: New drugs in the treatment of recurrent or metastatic cervical cancer. Crit Rev Oncol Hematol 2003;48:323-327.

31 Gadducci A, Tana R, Cosio S, Cionini L: Treatment options in recurrent cervical cancer (Review). Oncol Lett 2010;1:3-11.

32 Scatchard K, Forrest JL, Flubacher M, et al.: Chemotherapy for metastatic and recurrent cervical cancer. Cochrane Database Syst Rev 2012;(10):CD006469.

33 Monk BJ, Sill MW, McMeekin DS, et al.: Phase III trial of four cisplatin-containing doublet combinations in stage IVB, recurrent, or persistent cervical carcinoma: a Gynecologic Oncology Group study. J Clin Oncol 2009;27:4649-4655.
34 Moore DH, Tian C, Monk BJ, et al.: Prognostic factors for response to cisplatin-based chemotherapy in advanced cervical carcinoma: a Gynecologic Oncology Group Study. Gynecol Oncol 2010;116:44-49.

35 Kitagawa R, Katsumata N, Shibata T, et al.: Paclitaxe plus carboplatin versus paclitaxel plus cisplatin in metastatic or recurrent cervical cancer: the open-label randomized phase III trial JCOG0505. J Clin Oncol 2015; 33:2129-2135.

36 Minion LE, Bai J, Monk BJ, et al.: A Markov model to evaluate cost-effectiveness of antiangiogenesis therapy using bevacizumab in advanced cervical cancer. Gynecol Oncol 2015; 137:490-496.

37 Tewari KS, Sill MW, Long HJ, et al.: Improved survival with bevacizumab in advanced cervical cancer. $\mathrm{N}$ Engl J Med 2014;370:734-743. 